\title{
Metronomic oral vinorelbine in patients with advanced non-small cell lung cancer progressing after nivolumab immunotherapy: a retrospective analysis
}

\author{
Vittorio Gebbia ${ }^{1,2}$, Marco Maria Aiello33, Giuseppe Banna ${ }^{4}$, Giusi Blanco ${ }^{5}$, Livio Blasi ${ }^{6}$, Nicolò Borsellino ${ }^{7}$, Dario Giuffrida5 ${ }^{5}$ Mario Lo Mauro7, \\ Gianfranco Mancuso ${ }^{1}$, Dario Piazza ${ }^{8}$, Giuseppina Savio ${ }^{6}$, Hector Soto Parra ${ }^{3}$, Maria Rosaria Valerio ${ }^{9}$, Francesco Verderame ${ }^{10}$ and \\ Paolo Vigneri ${ }^{1}$
}

\author{
${ }^{1}$ Medical Oncology Unit, La Maddalena Clinic for Cancer Medical Oncology, Palermo 90100, Italy \\ ${ }^{2}$ PROMISE Department, University of Palermo, Palermo 90100, Italy \\ ${ }^{3}$ Policlinico-Vittorio Emanuele, Università di Catania, Catania 95100, Italy \\ ${ }^{4}$ Medical Oncology Unit, Ospedale Cannizzaro, Catania 95100, Italy \\ ${ }^{5}$ Medical Oncology Unit, IOM, Catania 95100, Italy \\ ${ }^{6}$ Medical Oncology Unit, ARNAS Civico, Palermo 90100, Italy \\ ${ }^{7}$ Medical Oncology Unit, Ospedale Buccheri La Ferla, Palermo 90100, Italy \\ ${ }^{8}$ Fondazione GSTU, Palermo 90100, Italy \\ 9Medical Oncology Unit, AOUP P. Giaccone, Palermo 90100, Italy \\ ${ }^{10}$ Medical Oncology Unit, Ospedale Cervello/Villa Sofia, Palermo 90100, Italy
}

\section{Abstract}

Purpose: The availability of immune checkpoint inhibitors has deeply changed the therapeutic scenario of patients with advanced non-small cell lung cancer (NSCLC). Up until now, chemotherapy still represents the first-line treatment for patients with advanced NSCLC not harbouring genetic mutations or lacking high expression of programmed death ligand even if the addition of immunotherapy to first-line chemotherapy has recently been shown to improve clinical outcome. We carried out a multi-institutional retrospective analysis on third-line chemotherapy with metronomic oral vinorelbine (VNR) in a series of patients with metastatic NSCLC pre-treated with first-line chemotherapy and second-line immunotherapy.

Patients and methods: Thirty patients with metastatic NSCLC with progressive disease after first-line chemotherapy and subsequent immunotherapy were treated with metronomic oral VNR continuously at the fixed dose of $30 \mathrm{mg}$ three times per week.

Results: A partial response was achieved in 4 patients (13.3\%), while 10 patients (33.3\%) displayed disease stabilisation for an overall disease control rate of $46.7 \%$. Median progression-free survival was 3.9 months (range 1-13 months) and median OS reached 8.1 months (range 4.0-24.0+ months) with a 12-month survival rate of $22 \%$.

Conclusion: Oral metronomic VNR appears to be active and safe in patients with metastatic NSCLC in progression after first-line chemotherapy and second-line immunotherapy. The results reported, although from a limited sample, may suggest its use for long-term stabilisation of the disease with good patient compliance.

Keywords: oral vinorelbine, metronomic therapy, non-small cell lung cancer, nivolumab
Correspondence to: Vittorio Gebbia Email: vittorio.gebbia@gmail.com

ecancer 2020, 14:1113

https://doi.org/10.3332/ecancer.2020.1113

Published: $29 / 09 / 2020$

Received: 23/04/2020

Publication costs for this article were supported by ecancer (UK Charity number 1176307).

Copyright: $($ ) the authors; licensee ecancermedicalscience. This is an Open Access article distributed under the terms of the Creative Commons Attribution License (http:// creativecommons.org/licenses/by/3.0), which permits unrestricted use, distribution, and reproduction in any medium, provided the original work is properly cited. 


\section{Introduction}

The availability of immune checkpoints inhibitors, such as nivolumab and pembrolizumab, has deeply changed the therapeutic scenario for patients with advanced non-small cell lung cancer (NSCLC) [1]. Up until now, platinum-based chemotherapy doublets still represent the first-line treatment of patients with advanced NSCLC not harbouring genetic alterations of oncogenic drivers or lacking high expression of the programmed death ligand even if the addition of immunotherapy to fist-line chemotherapy has recently been shown to improve clinical results $[2,3]$. Fit patients progressing after immunotherapy are often treated with subsequent lines of chemotherapy in daily clinical practice, but reliable scientific data in this disease setting are limited. Preliminary evidence has suggested that the immunotherapy pre-treatment improves response to subsequent chemotherapy in various oncological settings [4]. Indeed, a high objective response rate to third-line docetaxel (29\%) has been recently reported in a retrospective study in patients with advanced NSCLC pretreated with platinum-based chemotherapy and immunotherapy as first- and second-line treatments, respectively, suggesting a priming effect of previous immunotherapy [5].

Among chemotherapeutic agents employed in advanced NSCLC vinorelbine (VNR) has shown good anti-tumour activity and acceptable safety both after intravenous and oral administration [6, 7]. In the last decade, oral VNR has also been tested as single agent or in combination with platinum compounds in some phase I-II trials on a metronomic schedule, i.e., the administration of prolonged, non-stop low doses of otherwise active drugs [8-11]. In these studies, a flat dose in the range of 30-50 mg three times per week has been identified as the optimal schedule in terms of tolerability. The metronomic approach is considered intriguing due to its anti-angiogenic effect and the possible synergy with molecularly-targeted agents and immune checkpoints inhibitors [12-14]. Moreover, lack of major side-effects while preserving anti-neoplastic activity makes this schedule highly attractive for the management of heavily pretreated patients or elderly/frail ones [15-17].

In this paper, we report a multi-institutional study on third-line chemotherapy with oral metronomic VNR in a series of patients with metastatic NSCLC pretreated with firs-line chemotherapy and second-line immunotherapy.

\section{Material and methods}

\section{Study population}

Patients included in this analysis had to fulfil the following eligibility criteria: histologically confirmed diagnosis of metastatic NSCLC according to the TNM classification version 7.0 [18]; performance status on the Eastern Cooperative Oncology Group (ECOG) scale of 0-2; measurable disease according to the RECIST criteria [19]; progressive disease after second-line therapy with immune checkpoints inhibitors; adequate renal and hepatic functions; computed tomography scan and or CNS magnetic resonance imaging available for radiological review.

\section{Treatment schedule}

Oral VNR was given continuously at the fixed dose of $30 \mathrm{mg}$ three times per week (Monday, Wednesday and Friday) 30 minutes after a meal. Antiemetic therapy with oral ondansetron was given 1 hour before VNR. Four weeks of treatment were considered as one treatment cycle. The therapy was continued until progression, patient refusal or unacceptable toxicity. Complete blood cell counts and serum chemistry were obtained every 2 weeks.

\section{Design and statistics}

The aim of the study was to assess the activity and toxicity of metronomic oral VNR in a series of unselected patients with metastatic NCSLC after immunotherapy failure in patients progressing after first-line chemotherapy. The size of the statistical sample was not preprogrammed due to the descriptive nature of the analysis. After communication to the Ethical Committee, all clinical information for each eligible individual patient were retrospectively collected employing an anonymous electronic case report form with data directly tabulated on a central server. Objective responses according to the RECIST criteria were reported as absolute numbers and their relative rates; the sum of partial responses 
and disease stabilisation lasting more than 12 weeks was considered as disease control rate. Progression-free survival (PFS) and overall survival (OS) curves were calculated employing the Kaplan-Meier method. PFS was defined as the time from the beginning of metronomic oral VNR until objective neoplastic progression or death, whichever occurred first. Overall survival was defined as the time interval between the start of treatment and death or last follow-up contact. Side effects were recorded according to the Common Terminology Criteria for Adverse Events v 4.3, which is routinely used at all participating institutions, and was carried out by each investigator in their own institution.

\section{Results}

\section{Patient population}

Databases containing data from 249 advanced NSCLC patients treated in the last 4 years with second-line immune checkpoint inhibitors were queried for the use of metronomic oral VNR. Overall, 30 patients with metastatic NSCLC progressing after first-line chemotherapy and subsequent immunotherapy received oral VNR on a metronomic schedule in eight medical oncology units in Sicily. Table 1 describes the main clinical and demographic characteristics of subjects included in this analysis. Briefly, there were 23 males $(77 \%)$ and 7 females $(23 \%)$ with a median age of 69 years (range 49-80) and a median ECOG performance status of 1. Seventy percent of patients had an adenocarcinoma, while $27 \%$ were diagnosed with squamous cell carcinoma. Most patients (94\%) received first-line chemotherapy with platinum-based doublets, while two individuals had single-agent gemcitabine. All patients received immunotherapy with nivolumab (97\%) or pembrolizumab (3\%).

\section{Clinical outcomes}

As shown in Table 2, 4 patients (13.3\%) achieved a partial response and $10(33.3 \%)$ attained disease stabilisation with an overall disease control rate of $46.7 \%$. Sixteen patients (53.3\%) progressed. Median PFS was 3.9 months (range 1-13 months) and median OS reached 8.1 months (range 4.0-24.0+ months) with a 22\% 12-month survival rate (Figure 1). We found no correlation between objective response rates to VNR and any specific clinical feature. Two thirds of the individuals included in this study received a subsequent line of treatment.

\section{Safety}

Metronomic oral VNR was well tolerated. Overall, 116 cycles of chemotherapy were delivered. Grade 3 toxicities were rare with two patients developing anaemia that required blood transfusion, and one patient with transitory grade 3 neutropenia. Grades 1-2 fatigue and diarrhoea were reported in 8 (27\%) and 7 (23\%) cases, respectively. Grade 1 neutropenia was reported in five patients (175) but required no further treatment. Four patients (13\%) complained of grade 1 constipation. Oral VNR was delayed by at least 1 week in 9 patients (30\%).

\section{Discussion}

In the last decade, the administration of oral chemotherapy on a metronomic schedule has raised considerable interest among medical oncologists due to its preclinical rationale and clinical activity and safety [20]. Based on these data and on the observed efficacy of third-line chemotherapy after immunotherapy as compared to historical data [5], we carried out an analysis on the activity and tolerability of third-line chemotherapy with metronomic oral VNR in in patients with metastatic NSCLC pretreated with first-line chemotherapy and second-line immunotherapy.

We report a partial response in $13.3 \%$ of patients with disease stabilisation in $33.3 \%$ of our cohort with an overall disease control rate of 46.7\%. Median PFS was 3.9 months (range 1-13 months) and median OS reached 8.1 months (range 4.0-24.0+ months) with a 12-month survival rate of $22 \%$ (Figure 1). These findings are not surprising as other studies failed to identify correlations between patient outcomes and metronomic oral VNR pharmacokinetics [21, 22]. 
Table 1. Patient clinical and demographic characteristics.

\begin{tabular}{|c|c|c|c|}
\hline & & Number of patients & Percent \\
\hline & & 30 & 100 \\
\hline Age (years) & Median & 69 & \\
\hline Sex & Male & 23 & 77 \\
\hline Sex & Female & 7 & 23 \\
\hline \multirow[t]{2}{*}{ Smoke } & Never smoker & 8 & 27 \\
\hline & Former smoker & 22 & 73 \\
\hline \multirow[t]{3}{*}{ Histology } & Adenocarcinoma & 21 & 70 \\
\hline & Squamous cell & 8 & 27 \\
\hline & Large cell & 1 & 3 \\
\hline \multirow[t]{2}{*}{ EGFR status } & Wild type & 26 & 87 \\
\hline & Mutant & 4 & 13 \\
\hline ALK & Wild-type & 30 & 100 \\
\hline \multirow[t]{3}{*}{ Performance status } & ECOG 0 & 4 & 13 \\
\hline & ECOG 1 & 24 & 80 \\
\hline & ECOG 2 & 2 & 7 \\
\hline \multirow[t]{7}{*}{ Disease sites } & Lung & 27 & 90 \\
\hline & Nodes & 9 & 30 \\
\hline & Brain & 8 & 27 \\
\hline & Bone & 9 & 30 \\
\hline & Pleura & 2 & 6 \\
\hline & Liver & 4 & 13 \\
\hline & Peritoneum & 1 & 3 \\
\hline \multirow[t]{6}{*}{ Previous treatments } & First-line chemotherapy & 30 & 100 \\
\hline & Cisplatin-based & 28 & 94 \\
\hline & Single-agent & 2 & 6 \\
\hline & Second-line therapy & 30 & 100 \\
\hline & Nivolumab & 29 & 97 \\
\hline & Pembrolizumab & 1 & 3 \\
\hline
\end{tabular}

Table 2. Clinical outcomes.

\begin{tabular}{|l|c|c|}
\hline & $\mathbf{N}^{\circ}$ & $\%$ \\
\hline Enrolled patients & 30 & 100 \\
\hline Partial response & 4 & 13.3 \\
\hline Stable disease & 10 & 33.3 \\
\hline CBR & 14 & 46.7 \\
\hline Progressive disease & 16 & 53.3 \\
\hline
\end{tabular}




\section{Progression-free and overall survival}

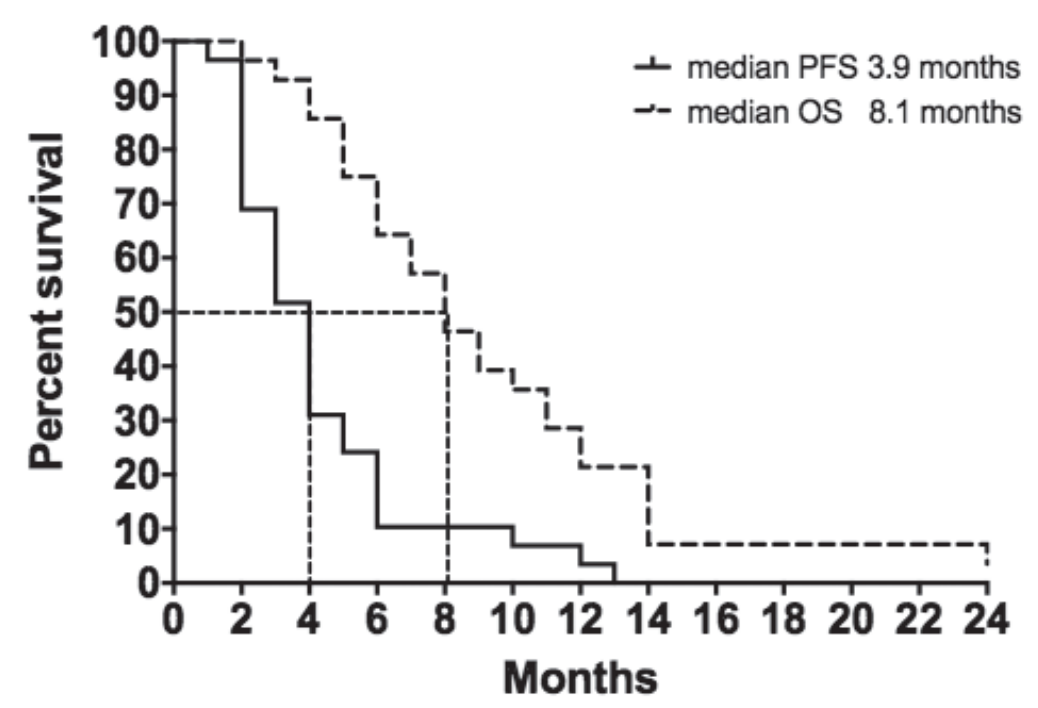

Figure 1. Progression free and overall survival.

Oral metronomic VNR at the dose of $50 \mathrm{mg} /$ day three times/week has been employed as first-line therapy in elderly and/or unfit patients with interesting results. Camerini et al [23] reported a series of 43 patients achieving a $18.6 \%$ overall response rate and a disease control rate of $58.1 \%$ with a median time to progression of 5 months (range 2-21) and median overall survival of 9 (range 3-29) months with limited grade 3 side-effects. Similar data were also reported in another series of 66 patients treated with the same VNR schedule [24]. Banna et al [15] employed fist-line metronomic oral VNR at the dose of $30 \mathrm{mg} /$ day in a series of 50 elderly/unfit patients with an overall disease control rate of $32 \%, 44 \%$ and $26 \%$ in first and subsequent lines, respectively. Median OS and PFS were 7.3 months and 2.7 months, respectively.

The activity and safety of metronomic oral VNR in second and subsequent lines has been described in three studies. The Hellenic Oncology Research Group reported a series of 26 patients with advanced NCSLC achieving an $11 \%$ overall response rate, a median PFS of 2.2 months and a median overall survival of 9.4 months with a 1-year survival rate of $30 \%$ [25]. Major side-effects were represented by grade 3 fatigue (11\%), neutropenia (8\%-24\%) and febrile neutropenia in 11\%. A Chinese study reported an $8 \%$ partial response rate and $42 \%$ stable disease rate for a disease control rate of $50 \%$ in a series of 26 patients [26]. The median number of treatment cycles received was 2 (range $1-8$ ), while median PFS was 2 months with a median follow-up time of 4 months (range 2-12). Good performance status (ECOG 1) was associated with longer PFS as compared to patients with ECOG 2 performance status, while no correlation was observed with sex, age, smoking status and histology. Major toxicity was grade 3 neutropenia in less than $10 \%$ of cases.

Finally, a multi-centre international retrospective real world analysis reported a series of 270 patients with advanced NCSLC with important co-morbid disease treated with oral metronomic VNR as first-, second- or third-line therapy [27]. The 38 patients treated with third-line oral metronomic VNR achieved a TTP of 4.0 months (range 1-19) months and a median OS of 6.5 months (range 2-29). The treatment was well tolerated with grade 3 neutropenia and fatigue observed in $2 \%$ of cycles. A significant percentage of patients achieved prolonged disease control and therefore continued their oral VNR. Moreover, patients treated with subsequent immunotherapy after first-line metronomic oral VNR achieved an OS that was 14 months longer than that of the entire recruited population. Authors speculated on the possible association of these results with the anti-angiogenic and/or immune stimulating properties of the metronomic approach. 


\section{Conclusion}

Our findings in an unselected patient population, although retrospective and in a small sample, suggest that oral metronomic VNR is an active and well-tolerated treatment for patients with metastatic NSCLC progressing after second-line immunotherapy. In our opinion, the good disease control rate achieved as a third-line treatment and good tolerability suggest that this pharmacological approach is a reasonable therapeutic option in this clinical setting.

\section{Conflicts of interest disclosure}

The author reports no conflicts of interest in this work.

\section{Authors' contributions}

Study concepts and design: VG; data acquisition and analysis: VG, DP; manuscript preparation, editing, and review: all authors.

\section{Funding}

Not applicable.

\section{References}

1. Verma V, Sprave T, and Haque W, et al (2018) A systematic review of the cost and cost-effectiveness studies of immune checkpoint inhibitors Immunother Cancer 23 128-142 https://doi.org/10.1186/s40425-018-0442-7

2. Planchard D, Popat S, and Kerr K, et al (2019) Metastatic non-small cell lung cancer: ESMO Clinical Practice Guidelines for diagnosis, treatment and follow-up Ann Oncol 30 1-3 https://doi.org/10.1093/annonc/mdy474

3. Langer CJ, Gadgeel SM, and Borghaei H, et al (2016) Carboplatin and pemetrexed with or without pembrolizumab for advanced, non-squamous non small- cell lung cancer: a randomised, phase 2 cohort of the open-label KEYNOTE- 021 study Lancet Oncol 17 1497-1508 https://doi.org/10.1016/S1470-2045(16)30498-3 PMID: 27745820 PMCID: 6886237

4. Emens LA and Middleton G (2015) The interplay of immunotherapy and chemotherapy: harnessing potential synergies Cancer Immunol Res 3 436-443 https://doi.org/10.1158/2326-6066.CIR-15-0064 PMID: 25941355 PMCID: 5012642

5. Schvartsman G, Peng SA, and Bis G, Lee JJ, et al (2017) Response rates to single-agent chemotherapy after exposure to immune checkpoint inhibitors in advanced non-small cell lung cancer Lung Cancer 112 90-95 https://doi.org/10.1016/j.lungcan.2017.07.034 PMID: 29191606

6. Gralla RJ, Gatzemeier U, and Gebbia V, et al (2007) Oral vinorelbine in the treatment of non-small cell lung cancer: rationale and implications for patient management Drugs 67 1403-1410 https://doi.org/10.2165/00003495-200767100-00003 PMID: 17600389

7. Gebbia V, Galetta D, and Lorusso V, et al (2008) Cisplatin plus weekly vinorelbine versus cisplatin plus vinorelbine on days 1 and 8 in advanced non-small cell lung cancer: a prospective randomized phase III trial of the G.O.I.M. (Gruppo Oncologico Italia Meridionale) Lung Cancer 61 369-377 https://doi.org/10.1016/j.lungcan.2008.01.010 PMID: 18308419

8. Briasoulis E, Aravantinos G, and Kouvatseas G, et al (2013) Dose selection trial of metronomic oral vinorelbine monotherapy in patients with metastatic cancer: a hellenic cooperative oncology group clinical translational study BMC Cancer 29(13) 263-274 https://doi. org/10.1186/1471-2407-13-263 
9. Barbolosi D, Ciccolini J, and Meille C, et al (2014) Metronomics chemotherapy: time for computational decision support Chemother Pharmacol 74 647-652 https://doi.org/10.1007/s00280-014-2546-1

10. Guetz S, Tufman A, and von Pawel J, et al (2017) Metronomic treatment of advanced non-small-cell lung cancer with daily oral vinorelbine-a Phase I trial Targets Ther 10 1081-1089 https://doi.org/10.2147/OTT.S122106

11. Pallis AG, Chandrinos V, and Pavlakou G, et al (2011) A multicenter phase I trial of metronomic oral vinorelbine plus cisplatin in patients with NSCLC Cancer Chemother Pharmacol 67 1239-1245 https://doi.org/10.1007/s00280-010-1415-9

12. Sutiman N, Zhang Z, and Tan EH, et al (2016) Phase I study of oral vinorelbine in combination with erlotinib in advanced non-small cell lung cancer (NSCLC) using two different schedules PLoS One 11 e0154316 https://doi.org/10.1371/journal.pone.0154316 PMID: 27135612 PMCID: 4852941

13. Qin RS, Zhang ZH, and Zhu NP, et al (2018) Enhanced antitumor and anti-angiogenic effects of metronomic vinorelbine combined with endostar on Lewis lung carcinoma BMC Cancer 18 967-978 https://doi.org/10.1186/s12885-018-4738-2 PMID: 30305062 PMCID: 6180630

14. Orlandi P, Di Desidero T, and Salvia G, et al (2018) Metronomic vinorelbine is directly active on non small Cell lung cancer cells and sensitizes the EGFRL858R/T790M cells to reversible EGFR tyrosine kinase inhibitors Biochem Pharmacol 152 327-337 https://doi. org/10.1016/j.bcp.2018.04.011 PMID: 29660315

15. Banna GL, Camerini A, and Bronte G, et al (2018) Oral metronomic vinorelbine in advanced non-small cell lung cancer patients unfit for chemotherapy Anticancer Res 38 3689-3697 https://doi.org/10.21873/anticanres.12647 PMID: 29848729

16. D'Ascanio M, Pezzuto A, and Fiorentino C, et al (2018) Chemotherapy with vinorelbine produces clinical benefit and low toxicity in frail elderly patients affected by advanced non-small cell lung cancer Biomed Res Int 20186278403

17. Bilir C, Durak S, and Kızlkaya B, et al (2017) Efficacy of metronomic vinorelbine in elderly patients with advanced non-small-cell lung cancer and poor performance status Curr Oncol 24 e199-e204 https://doi.org/10.3747/co.24.3486 PMID: 28680287 PMCID: 5486392

18. Goldstraw P, Crowley J, and Chansky K, et al (2016) The IASLC Lung Cancer Staging Project: proposals for the revision of the TNM stage groupings in the forthcoming (seventh) edition of the TNM Classification of malignant tumours J Thorac Oncol 2 706-714 https://doi. org/10.1097/JTO.0b013e31812f3c1a

19. Eisenhauer EA, Therasse P, and Bogaerts J, et al (2009) New response evaluation criteria in solid tumours: revised RECIST guideline (version 1.1) Eur J Cancer 45 228-247 https://doi.org/10.1016/j.ejca.2008.10.026

20. Cazzaniga ME, Camerini A, and Addeo R, et al (2016) Metronomic oral vinorelbine in advanced breast cancer and non-small-cell lung cancer: current status and future development Future Oncol 12 373-387 https://doi.org/10.2217/fon.15.306

21. Gusella M, Pasini F, and Caruso D, et al (2018) Clinical outcomes of oral metronomic vinorelbine in advanced non-small cell lung cancer: correlations with pharmacokinetics and MDR1 polymorphisms Cancer Chemother Pharmacol https://doi.org/10.1007/s00280-0183751-0 [Epub ahead of print] PMID: 30542768

22. Pasini F, Barile C, and Caruso D, et al (2018) Oral metronomic vinorelbine (OMV) in elderly or pretreated patients with advanced non small cell lung cancer: outcome and pharmacokinetics in the real world Invest New Drugs 36 927-932 https://doi.org/10.1007/s10637018-0631-8 PMID: 29956056

23. Camerini A, Puccetti C, and Donati S, et al (2015) Metronomic oral vinorelbine as first-line treatment in elderly patients with advanced non-small cell lung cancer: results of a phase II trial (MOVE trial) BMC Cancer 15359 https://doi.org/10.1186/s12885-015-1354-2 PMID: 25943747 PMCID: 4424528

24. Mencoboni M, Filiberti RA, and Taveggia P, et al (2017) Safety off first-line chemotherapy with metronomic single-agent Oral vinorelbine in elderly patients with NSCLC Anticancer Res 37 3189-3194 PMID: 28551663 
25. Kontopodis E, Hatzidaki D, and Varthalitis I, et al (2013) A phase II study of metronomic oral vinorelbine administered in the second line and beyond in non-small cell lung cancer (NSCLC): a phase II study of the Hellenic Oncology Research Group J Chemother 25 49-55 https://doi.org/10.1179/1973947812Y.0000000050 PMID: 23433445

26. Yao S, Gu Y, and Zhang Y (2017) Efficacy and toxicity of metronomic oral vinorelbine in advanced non-small cell lung cancer after failure to multiple-lines treatments Zhongguo Fei Ai Za Zhi 20 737-740 PMID: 29167002 PMCID: 5973282

27. Camerini A, Banna GL, and Cinieri S, et al (2018) Metronomic oral vinorelbine for the treatment of advanced non-small cell lung cancer: a multicenter international retrospective analysis Transl Oncol published ahead of print. 